\title{
RELIABILITY OF FLUORESCENT CAMERA VERSUS VISUAL ASSESSMENT IN DIAGNOSIS OF OCCUSAL CARIOUS LESION IN ADULT PATIENTS: INVIVO DIAGNOSTIC STUDY
}

\author{
Shimaa Maher Zenhom Abd El Rahman*, Mona Ismail Riad** and Shereen Hafez Ibrahim***
}

\begin{abstract}
Aim: This study aimed to compare the diagnostic predictive values of a light induced fluorescence intra oral camera (VistaCam iX) versus visual assessment method (ICDAS-II) in identification of occlusal carious lesion.

Methodology: Occlusal surfaces of 199 molar teeth from 43 adult patients who fulfill the eligibility criteria were examined by two observers using two diagnostic methods. The teeth were firstly assessed visually according to the criteria of the International Caries Detection and Assessment System (ICDAS-II) then by fluorescence camera. Inter observer agreement was measured for ICDAS-II using Kappa and weighted Kappa Values and for the camera using inter class correlation (ICC). Correlation between methods was calculated using Spearman's rank correlation coefficient $\left(r_{s}\right)$ and the Receiver Operating Characteristic (ROC curve) between the tested methods was estimated.
\end{abstract}

Results: Significant positive correlation was found between the ICDAS-II and the camera measurements $\left(r_{s}=0.8739, \mathrm{P}<0.001\right)$. The area under the ROC curve was $(0.932, \mathrm{p}<0.001)$ indicating a great association between the two tested methods when selecting adequate cut-off values for the camera. There was a statistically significant agreement between the two observers for ICDAS-II values (Kappa $=0.8125$, weighted Kappa $=0.8558)$. Excellent inter examiner agreement was found between the two observers when using the FC, ICC $=0.9774$ (95\% CI, 0.789 to 0.889 ), $(\mathrm{p}<0.001)$.

Conclusion: The camera showed high reliability and agreement with ICDAS-II for initial occlusal caries detection. Sensitivity and specificity values for the camera varied depending on the cut-off value.

KEYWORDS: VistaCam iX Proof, Occlusal caries, ICDAS-II, Fluorescence camera, Initial caries, Visual examination.

* Master's Degree in Restorative and Esthetic Dentistry.

** Professor of Conservative dentistry, Faculty of Dentistry, Cairo University.

*** Assistant Professor of Conservative Dentistry, Faculty of Dentistry, Cairo University. 


\section{INTRODUCTION}

The main goal of modern dentistry is the early detection of incipient carious lesions. (Bader et al. 2002). The occlusal caries detection presents a challenge due to the complex morphology of the groove-fossa system and frequent presence of contaminants that might interfere with good diagnosis. The early detection of carious lesions is important in order to perform preventive and interceptive treatment strategies (Peycheva and Boteva. 2016).

Previous data indicate that visual examination is only effective in more pronounced stages, highlighting the need for reliable detection in the pre-cavitation stages of the carious process (Dąbrowski et al. 2019), For this reason, the search is on for more sensitive and specific tools, using new technologies, to help the practitioner to diagnose initial carious lesions as accurately as possible .

Recently, in order to improve the accuracy of non-cavitated occlusal caries detection, diagnostic tools have been introduced, which are based on physical properties such as tooth fluorescence on light of visual spectrum Vista cam (Achilleos et al. 2013)

Fluorescence assessment is based on the principle that chromophores in the dental enamel and dentine cause auto-fluorescence that is reduced by demineralization. Chromophores in caries lesions and bacteria also cause fluorescence that can be quantified and measured by subtracting the fluorescence of a healthy tooth surface from that of carious tooth surface (Stoll et al. 2015).

The null hypothesis tested is that there is no difference between light induced fluorescence camera and visual assessment method in identification of occlusal carious lesion.

Aim of the study: This study was conducted to compare the diagnostic predictive values of a light induced fluorescence camera versus visual assessment method in identification of occlusal carious lesions.

\section{MATERIAL AND METHODS}

\section{Patient selection}

A total of 43 patients involved in the study. Each patient had at least one non-cavitated occlusal carious lesion in each arch (maxilla and mandible) in the first and /or second molar, resulting in a total of 199 examined teeth $(\mathrm{N}=199)$. Each lesion had been evaluated by two diagnostic methods (D), where $D_{1}$, represents visual assessment method (ICDAS II criteria) and $\mathrm{D}_{2}$ represents light induced fluorescence camera.

- Inclusion Criteria of teeth:

1. Teeth with ICDAS codes from 0 - 2 (JablonskiMomeni et al. 2016).

- Exclusion criteria of teeth:

1. Teeth with any kind of malformations, such as fluorosis, enamel hypoplasia, amelogenesis imperfecta, hypo-mineralization and / or frank occlusal cavitation (Diniz et al. 2012).

2. Teeth with intrinsic or extrinsic staining, sealants and restorations (Diniz et al. 2012).

3. Teeth having large carious lesions on smooth and proximal surfaces (Diniz et al. 2012).

4. Third molars are not included (Presoto et al. 2017).

\section{Armamentarium used in this study:}

1. Light induced fluorescence intraoral camera (LF): (Vista-Cam iX, DürrDental, BietigheimBissingen, Germany)

2. Mirror: (high definition dental mirror, $\mathbf{H u}-$ Friedy, USA)

\section{Criteria of examiners:}

All examinations were performed by two examiners: 


\section{Examiner 1 (co-supervisor)}

2. Examiner 2 (The researcher)

Examiner 1 (co-supervisor) had already previous experience using ICDAS-II criteria and fluorescence-based caries detection camera.

The examiner 2 was trained and calibrated in ICDAS-II and in using the fluorescence camera by the experienced examiner 1 . The training included theoretical instruction and practical exercise, following this the examiner 1 had a practical examination on extracted teeth that represented the different stages of the ICDAS-II scores, then scanned them by the fluorescent camera, and any errors made were immediately discussed and resolved (Jablonski-Momeni et al. 2008) and (Jablonski-Momeni et al. 2013).

\section{Randomization and assignment of interventions}

No randomization was done as all patients were evaluated using both intervention/Comparator assessment methods by both examiners.

\section{Clinical examination}

Each patient was diagnosed by both the assessment methods twice, one by each examiner (in separate cabins), and the examiners were unaware of each other's results. The investigation sites were recorded and identified by simple drawing dots to guide lesion location (Marczuk-Kolada et al.2020). The highest ICDAS score in each occlusal surface was recorded as the investigation site (Jablonski Momeni et al.2016). The surfaces chosen for scoring were identical for both methods. The illumination, cleaning and drying procedures were the same in the diagnosis of all patients (Bhoopathi et al. 2017).

\section{Visual examination}

Prior to the visual examinations, polishing of all the teeth was done to eliminate the surface biofilm and deposits by using a low-speed handpiece with a rotating bristle brush and water (Diniz et al. 2011). then they were examined visually with the aid of the light of dental unit lamp, air-water spray and the dental mirror

First the examiner analyzed the teeth when wet, then when dry, by drying the teeth for 5 seconds using a triplex syringe using the classification system (ICDAS-II) which includes six codes (Al-Khalifa et al. 2016)

- Zero: No or slight change in enamel translucency after prolonged air drying

- One: First visual change in enamel (seen after prolonged air drying or restricted to within the confines of a pit or fissure

- Two: Distinct visual changes in enamel

- Three: Localized enamel breakdown in opaque or discolored enamel (without visual signs of dentinal involvement)

- Four: Underlying dark shadow from dentine

- Five: Distinct cavity with visible dentine

- Six: Extensive distinct cavity with visible dentine (involving more than half of the surface)

\section{Fluorescence examination}

After the examiners performed the visual examination, the same participants were subjected to a new assessment method using the Vista Cam fluorescence camera (FC). The examiners captured images of the occlusal surfaces of teeth using the fluorescence induced head (Proof interchangeable head) accordance with the manufacturer's instructions. Before the use, the head of the intraoral camera was placed inside a special hygienic protective wrap provided by the manufacturer. Firstly, the head of the camera was positioned perpendicularly on the occlusal surface of the teeth (with a spacer connected onto the head) under isolation with cotton rolls and suction tip and after tooth drying with a triplex air syringe for 15 seconds (Jablonski-Momeni et al. 2016). By pressing the 
control ring of the device an image was captured. Then the image was analyzed by the special software (DBSWIN). This software quantifies the red and green components of fluorescence in each region of the tooth and produces a digital image that shows lesions in different color shades with numerical value between 0 and 3 predicting the depth and extent of the carious demineralization, higher values indicate more severe carious lesions as presented in the manufacturer's color code in Table (1) (Stoll et al. 2015).However, the current study used clinical cutoffs obtained in previous in-vivo clinical study by Presoto et al. (2017) that presented in Table (2).

TABLE (1) The manufacturer's color code of (Vista-Cam iX).

\begin{tabular}{|c|c|}
\hline Healthy tooth enamel & $0: 1$ (Green) \\
\hline Early stages of enamel caries & $1: 1.5$ (Blue) \\
\hline Deep enamel caries & $1.5: 2$ (Red) \\
\hline Dentine caries & $2: 2.5$ (Orange) \\
\hline Deep dentine caries & $>2.5$ (Yellow) \\
\hline
\end{tabular}

TABLE (2) Score scale of Vista Cam: (Presoto et al. 2017)

\begin{tabular}{|c|l|c|c|}
\hline $\begin{array}{c}\text { Score } \\
(\mathrm{mm})\end{array}$ & \multicolumn{1}{|c|}{ Description } & $\begin{array}{c}\text { Presence } \\
\text { of caries }\end{array}$ & $\begin{array}{c}\text { Color } \\
\text { code }\end{array}$ \\
\hline $0 \leq \mathrm{x}<1$ & Sound enamel & Absent & Green \\
\hline $1 \leq \mathrm{x}<1.5$ & $\begin{array}{l}\text { Initial caries, beginning } \\
\text { enamel caries }\end{array}$ & Present & Blue \\
\hline $1.5 \leq \mathrm{x}<2$ & $\begin{array}{l}\text { Enamel caries to enamel- } \\
\text { dentin limit }\end{array}$ & Present & Red \\
\hline $2 \leq \mathrm{x}<2.5$ & Dentin caries & Present & Orange \\
\hline $\mathrm{x} \geq 2.5$ & Deep dentin caries & Present & Yellow \\
\hline
\end{tabular}

Finally, the image was saved, and the values of each investigation site were recorded. If a fluorescence value of visually specified investigation site was not displayed automatically on the image by the software, the investigator selected this site manually on the image, and so, the value for this site was analyzed by the software and displayed on the image (Jablonski-Momeni et al. 2016).

\section{Statistical analysis}

Categorical data were presented as frequencies and percentages and were analyzed using chi square test. Numerical data of age were presented as mean, standard deviation (SD) and confidence interval values. Data were explored for normality by checking the data distribution, calculating the mean and median values and using KolmogorovSmirnov and Shapiro-Wilk tests. Data showed parametric distribution and were analyzed using independent t-test. Weighted kappa coefficient was used for reliability analysis. Paired t-test was used to determine the difference in agreement. The significance level was set at $\mathrm{P} \leq 0.05$ within all tests. Statistical analysis was performed with IBM $^{\circledR}$ SPSS $^{\circledR}$.

\section{RESULTS}

This study was held to compare the performance of fluorescence camera in detection of carious lesions with the traditional ICDAS-II method. In addition, inter-observer agreement measurements were computed to show the degree of reliability of both methods on molar teeth.

\section{Inter-Observer Agreement for ICDAS-II:}

There was a substantial to almost perfect agreement between the two observers where kappa, and weighted kappa values were 0.8125 , and 0.8558 respectively. It was a statistically significant agreement, $\mathrm{Kw}=0.8558$ (95\% CI, 0.789 to 0.889$)$, $(\mathrm{p}<0.001)$. 


\section{Inter-Observer Agreement for Fluorescence Camera}

There was an excellent agreement and consistency in the measurements of the camera which reflects a very high degree of reliability in using the Fluorescence camera in caries detection. This was a statistically significant level of agreement where $(\mathrm{ICC})=0.9774(95 \% \mathrm{CI}, 0.789$ to 0.889$)$, $(\mathrm{p}<0.001)$.

\section{Spearman's Rank Correlation between ICDAS II and Fluorescence Camera}

To measure the level of correlation between the measurements of both ICDAS II and Fluorescence camera, the spearman's rank correlation coefficient is computed for the measurements of the first operator from each method. The calculation resulted in an 0.8739 positive correlation value between the camera and ICDAS-II with P-Value < 0.001 which showed a strongly significant correlation between both methods that was positive, and direct.

The level of fluorescence camera agreement with the traditional ICDAS-II was evaluated using different measurements including sensitivity, and specificity of detecting caries in addition to overall accuracy, positive predictive value, and negative predictive value. All results are shown below in Table (3) where the ICDAS-II is considered as the ground truth method. Overall, the fluorescence camera showed a high level of agreement with the ICDAS-II in carious lesion detection with high sensitivity value of $94.92 \%$ for the carious lesion (positive class), the specificity is a little bit smaller to reach $87.65 \%$ to achieve an overall accuracy of $91.96 \%$. The positive and negative predictive values were $91.80 \%$ and $92.21 \%$ respectively showing a slightly better predictive value for sound teeth than the carious teeth.
TABLE (3): Cross Tabulation of Sound vs. Carious Detection Agreement between ICDAS-II scores and Fluorescence Camera in whole sample size.

\begin{tabular}{|c|c|c|c|}
\hline $\begin{array}{c}\text { Fluorescence } \\
\text { Camera }\end{array}$ & Sound $(<\mathbf{1})$ & $\begin{array}{c}\text { Carious } \\
(>\mathbf{= 1})\end{array}$ & All \\
\hline ICDAS II & \multicolumn{3}{|c|}{} \\
\hline Sound (=0) & 71 & 10 & $81(40.70 \%)$ \\
\hline Carious (>0) & 6 & 112 & $118(59.30 \%)$ \\
\hline All & $77(38.69 \%)$ & $122(61.31 \%)$ & 199 \\
\hline
\end{tabular}

\section{ROC (Receiver operator curve):}

The area under the ROC (AUC) was significantly high to reach 0.932 which indicates a great association between ICDAS-II and Florescence camera scores when selecting an adequate cut-off value for the camera.

\section{DISCUSSION}

The rationale of the current study was the importance of early detection of caries in order to decide whether to perform preventive or interceptive treatment strategies (Guerrieri et al. 2012).

In the literature there are several detection methods to detect initial caries on occlusal surfaces. Histological examination in in-vitro studies is considered the gold standard that can determines the extent of dental caries (Lino et al. 2015). However, a limitation of this in-vivo study was the impossibility of opening the teeth classified as non-carious and performing histological examination. Therefore, visual inspection by ICDAS-II was selected as the comparison standard.

However, initial carious lesions have generated most of the disagreements between examiners in several studies, and their evaluation demands more training and more time for examination (Bader et al. 2002). 
In diagnosis of caries with visual methods even when using detailed system as ICDAS-II, there is a degree of subjective evaluation; as it relies on the experience of the practitioner and due to difference in visual perception of different examiners (Jablonski-Momeni et al. 2009).

Therefore, other methods have been proposed for objective evaluation, So Hibst et al. (2001) has explored the implementation of fluorescence as an alternative diagnostic tool in the detection of occlusal caries hidden under a macroscopically sound surface, motivated by the poor sensitivity of visual inspection and radiography.

The second method used in this study was the light induced fluorescence intraoral camera (VistaCam- iX).

These fluorescence properties of the tooth tissues allow the Vista Cam iX fluorescent camera to differentiate between carious and non-carious stained fissures and help in detection of the so called "hidden caries" especially carious lesion under apparently intact tooth surface (Guerra et al. 2015).

The images produced by the Vista cam fluorescent camera have the advantage of being classified according to scores provided by the manufacturer, based on the extent of the carious lesion (JablonskiMomeni et al. 2013). This helps in standardizing the calibration of the device and the examiners which is important, as good reproducibility is the first step in obtaining consistent results from various examiners at various assessment periods.

The fluorescence camera allows image documentation; so this helps to monitor lesions and these images can be viewed at subsequent checkups and compared to the new findings, thus facilitate controlling the progression of lesions over time and could postpone invasive treatment that only would be performed when a lesion clearly shows to be progressive (Rodrigues et al. 2008).

The sample size had calculated 43 patients would be sufficient, for this study patients with at least one non cavitated occlusal caries lesion in each arch (maxilla and/or mandible) were selected; because caries clinical trials require large numbers of subjects (teeth) to get reliable results (Kingman and Selwitz 1997).

Only permanent molars were examined in this study because the molar teeth are the most significantly affected teeth by caries at $45 \%$. Regarding caries frequency, the occlusal fissures of the first and second maxillary molars demonstrated the highest caries frequencies at $52.7 \%$ and $60.9 \%$, respectively. While regarding caries rate, occlusal fissures of the first and second mandibular molar teeth showed the highest caries rates $(55.6 \%$ and $66.3 \%$, respectively), so this study was conducted on the occlusal surfaces of first and second permanent molar teeth (Demirci et al. 2010).

The occlusal surfaces of the molars were chosen to be diagnosed in the current study because they are still the most likely sites for the development of lesions, they also represent a challenge in their diagnosis due to their characteristic invaginated anatomy (Rodrigues et al. 2009)

Before visual examination, Scaling and precleaning of the teeth is performed with a rotating brush and water which is fundamental to the quality of diagnosis by both the direct visual examination and to the complementary diagnostic aids such as fluorescence-based techniques (Diniz et al. 2011).

No prophylactic pastes were used to avoid their possible influence on measurements made by fluorescence-based methods (Pontes et al. 2017).

The clinical examinations selected the highest ICDAS score site on each tooth surface to be the test site, because having numerous investigation sites on one tooth doesn't result in a statistically independent data (Jablonski-Momeni et al. 2016).

During visual examination, the examiners used high definition mirror which features a proprietary special coating that claimed by the manufacturer to provide superior brilliance and color for increased 
visibility in all dental procedures, also it is scratchresistant for product longevity.

All the examination procedures including illumination, cleaning and drying were the same in all patients; to allow standardized procedures and to avoid any discrepancy in the recording pattern (Bhoopathi et al. 2017).

Both examiners (Examiner 1 and Examiner 2) performed the procedures in separate cabins, each examiner had different assistant (Assistant 1 and Assistant 2), thus controls blinding by preventing exchange any information through the entire study period.

After visual examination the teeth were assessed by the fluorescent camera, the camera head was placed on the occlusal surface of the teeth using a spacer as claimed by manufacturer to provide stable position and fixed distance between the tip of the camera and the tooth in order to obtain reproducible images each time.

Examination and recording images by the camera was done under isolation with cotton rolls and suction tip and after tooth drying with a triplex air syringe for 15 seconds, as according to De Benedetto et al. (2011) a statistically significant increase in FC readings shown after drying for $15 \mathrm{~s}$. and specially in dehydrated teeth.

The results of Inter-Observer Agreement for ICDAS-II system is presented by kappa, and weighted kappa values which showed almost substantial to a perfect agreement between measurements of both observers.

This result might be due to the examiners had received more training on using the ICDAS-II scoring system before beginning of the actual study (Jablonski-Momeni et al. 2008), also the teeth had been examined by direct visual examination and not by indirectly via white light photographs. On the photographs, the teeth were mostly dried, whereas during visual examination the teeth can be visualized wet or dried, so ICDAS score 1 and 2 can be more easily differentiated. (Theocharopoulou and Lagerweij. 2015)

Rodrigues et al (2008) found that the Interexaminer values for the ICDAS II scoring system were 0.51 . The inferior results in the study can be explained by different clinical experiences between examiners and tolerance of the examiners at ICDASII criteria.

Almost all the studies showed adequate interexaminer reproducibility for the ICDAS scoring system with varying ranges of agreement. The variations among different studies could be explained by the subjective aspects involved in visual examination, such as clinical experience and knowledge of the examiners and vision precision of each examiner (Fung et al. 2004). Besides the differences in methodology and preparations before the study, also it is important to keep in mind that some investigations were performed in-vivo, and others in-vitro, and this could be an explanation of the different values of reproducibility found among the studies.

There was an excellent agreement and consistency in the measurements of the camera which reflects a very high degree of reliability in using the Fluorescence camera in lesion detection.

The high values of inter-examiner ICC that observed with the FC in the current study may be attributed to the training of the examiner about how to use the new diagnostic fluorescent camera and also it may be due to taking the reading of the exact examination site on the occlusal surface of each tooth every time the tooth is examined, guided by the simple drawing of the examination site on the occlusal surface of each tooth.

Many researches also confirmed the high level of reproducibility of the camera likewise confirmed in studies by Rodrigues et al. (2008), JablonskiMomeni et al. (2011), Novaes, et al. (2012) and De Benedetto et al. (2011). 
This finding was in dis-agreement with Dąbrowski et al. (2019) who found that the interobserver weighted kappa value for VistaCam was 0.54 , and this result is considered far from being satisfactory. This result may be due to the different biogenic apatite of the archaeological teeth examined in this study.

The current study showed a positive correlation between the measurements of both ICDAS II and Fluorescence camera. The high correlation between ICDAS-II and camera might be due to that both methods have the same idea and nearly follow the same score in initial caries diagnosis, as presented in the Table (4).

TABLE (4): Comparison between the scores of ICDAS-II and FC

\begin{tabular}{|l|l|l|}
\hline & ICDAS-II & Fluorescent Camera \\
\hline Sound & Score 0 & Score $0 \leq \mathrm{x}<1$ \\
\hline Initial enamel & Score 1 & Score $1 \leq \mathrm{x}<1.5$ \\
\hline Deep enamel & Score 2 & Score $1.5 \leq \mathrm{x}<2$ \\
\hline
\end{tabular}

This finding was in acceptance with JablonskiMomeni et al. (2012) who found high correlation between ICDAS-II and FC (rs 0.84) and the results of the study showed that the visual method and the fluorescence camera exhibited the highest correlation to the gold standard.

Seremidi et al. (2012) suggested that fluorescence methods for detection of occlusal caries in permanent teeth were not found to be significantly different and not better than visual methods and, in some cases, worse than them. This may be because of stain inclusion in the lesions, resulting in higher measurement values and consequently in higher rates of false positives.

This finding disagreed with Guerra et al. (2016) who found a fair correlation between the ICDASII and the fluorescence measurements; this could be explained by that the ICDAS-II method in certain cases might underestimate initial caries and overestimate deep caries.

The high sensitivity and specificity values of the camera found in the present study might be due to that; carious tissue and healthy tissue emit fluorescence at different intensities when excited by light at specific wavelengths; working in accordance with this principle, Vista Cam iX fluorescent camera can improve the accuracy of diagnosis of the socalled hidden caries, especially occlusal carious lesions underlying a macroscopically sound tooth surface, and so the device shows high sensitivity and specificity for caries detection (Guerra et al. 2015).

Similar findings were described by Presoto et al. (2017) and Schwendicke et al. (2015). MarczukKolada et al. (2020) also found that The VistaCam iX was characterized by a high sensitivity for the enamel threshold like the ICDAS II scoring system.

Unlike the previous studies Diniz et al. (2012) assessed occlusal surfaces by using the LF device (DIAGNOdent), LF pen(DIAGNOdent Pen), FC, (ICDAS) criteria and bitewing $(\mathrm{BW})$ radiographic methods, and found that FC had the lowest performances in helping detect lesions at the D1, as it found that the use of the optimal cutoffs for the FC worsened the sensitivity and accuracy values and improved specificity values at D1. Although the FC helped detect $99 \%$ of the enamel carious lesions correctly with the use of the manufacturer cutoffs, specificity and sensitivity values differed with the use of the optimal cutoffs, indicating that those cutoffs should be used carefully in clinical practice.

The different results of the studies in agreement values between ICDAS-II and the camera could be explained by the cutoff values used, as the clinical performance of fluorescence-based detection methods depends mainly on the cutoff used. Manufacturers offer cutoffs differ than those obtained in in-vitro and in-vivo studies, which may clarify variations of results found in the literature, which can confuse clinicians who are trying to 
decide the best treatment (Diniz et al .2012) .Cutoff values tend to differ according to the type of the study (in vitro, in vivo) and the teeth (primary, permanent). (Jablonski-Momeni et al. 2016)

The software highlighted the carious lesions in different colors and defined their extent on a scale from 0 to $5: 1.0$ to 1.5 (blue) indicated beginning enamel caries, 1.5 to 2.0 (red) indicated deep enamel caries, 2.0 to 2.5 (orange) indicated dentin caries, and 2.5 to 5.0 (yellow) indicated deep dentin caries. These cutoffs should be interpreted carefully, as a 0.1 variation could cause trouble when making a treatment decision. (Rodrigues et al. 2008)

The importance of cutoffs means that a small change in the reading could be the difference between performing an operative intervention or prevention, which is a dangerous aspect of these devices if they are used throughout the decisionmaking process. (Diniz et al .2009) and (Rodrigues et al. 2008)

In the present study the Receiver operator curve (ROC) for different cut-off points of the Fluorescent camera showed a great association between ICDAS II and Florescent camera scores. The advantages of ROC curve are: (a) it includes several cut-off points; (b) it shows the relationship between the sensitivity and specificity.

Initial in-vitro studies using ICDAS-II for detection of occlusal lesions showed lower overall performance of ICDAS-II (AUC between 0.73-0.88) (Jablonski-Momeni et al. 2008) and (Rodrigues et al. 2008). Later studies showed an increase of ICDAS-II performance, sensitivity, and specificity values which might be explained by the enhanced training in this system (Jablonski-Momeni et al. 2017).

This result is in dis-agreement with Dąbrowski et al. (2019) who found the area under curve (AUC) for vista cam was 54.2; however, this result may be due to the different structure of the archaeological teeth examined in this study.

\section{CONCLUSIONS}

1. The intra oral fluorescent camera exhibited high reliability for initial occlusal caries detection like ICDAS-II scores.

2. Sensitivity and specificity values for the camera varied depending on the cut-off value used.

3. The fluorescent camera showed good diagnostic performance with high Sensitivity and specificity values.

4. The performance of fluorescence camera was not significantly different and not better than ICDAS-II for the detection of non-cavitated carious lesions.

5. A short training of dentists in the use the ICDASII system and the fluorescent camera resulted in satisfactory inter-examiner reliability for the analysis of non-cavitated occlusal lesions.

\section{REFERENCES}

- Achilleos, E.-E., Rahiotis, C., Kakaboura,A., \& Vougiouklakis, G. (2013): Evaluation of a new fluorescence-based device in the detection of incipient occlusal caries lesions. Lasers in medical science, 28 (1), 193-201.

- Al-Khalifa, K.S. (2016): Use of the International Caries Detection and Assessment System by Dental Students at the University of Dammam, Saudi Arabia. The Saudi Journal for Dental Research, 7(1), 38-44.

- $\quad$ Bader, J. D., Shugars, D. A., \& Bonito, A. J. (2002): A systematic review of the performance of methods for identifying carious lesions. Journal of public health dentistry, $62(4), 201-213$.

- $\quad$ Bhoopathi, P. H., Patil, P. U., Kamath, B. V., Gopal, D., Kumar, S., \& Kulkarni, G. (2017): Caries detection with icdas and the who criteria: A comparative study. Journal of Clinical \&Diagnostic Research, 11(12), 9-12

- Dąbrowski, P., Grzelak, J., Kulus, M. and Staniowski, T., (2019): Diagnodent and VistaCam may be unsuitable for the evaluation of dental caries in archeological teeth. American j ournal of physical anthropology, 168(4), 797-808.

- De Benedetto, M. S., Morais, C. C., Novaes, T. F., de Almeida Rodrigues, J., Braga, M. M., \& Mendes, F. M. 
(2011): Comparing the reliability of a new fluorescence camera with conventional laser fluorescence devices in detecting caries lesions in occlusal and smooth surfaces of primary teeth. Lasers in medical science, 26 (2), 157-162.

- $\quad$ Demirci, M., Tuncer, S., \& Yuceokur, A. A. (2010): Prevalence of caries on individual tooth surfaces and its distribution by age and gender in university clinic patients. European journal of dentistry, 4(03), 270-279.

- Diniz, M. B., Rodrigues, J. A., Hug, I., De Cássia Loiola Cordeiro, R., \& Lussi, A. (2009): Reproducibility and accuracy of the icdas-ii for occlusal caries detection. Community dentistry and oral epidemiology, 37(5), 399-404.

- Diniz, M., Sciasci, P., Rodrigues, J., Lussi, A., \& Cordeiro, R. d. C. L. (2011): Influence of different professional prophylactic methods on fluorescence measurements for detection of occlusal caries. Caries research, 45 (3), 264-268.

- Diniz, M. B., Boldieri, T., Rodrigues, J. A., Santos-Pinto, L., Lussi, A., \& Cordeiro, R. C. (2012): The performance of conventional and fluorescence-based methods for occlusal caries detection: an in vivo study with histologic validation. The Journal of the American Dental Association, 143 (4), 339-350.

- Fung, L., Smales, R., Ngo, H., \& Mount, G. (2004): Diagnostic comparison of three groups of examiners using visual and laser fluorescence methods to detect occlusal caries in vitro. Australian dental journal, 49(2), 67-71.

- $\quad$ Guerra, F., Mazur, M., Rinado, F., Corridore, D., Salvi, D., Pasqualotto, D., \& Ottolenghi, L. (2015): New diagnostic technolog ur, M., Rinaldo, F., Mergè, F., Pasqualotto, D. Ottolenghi, L. (2016): Early caries detection: comparison of two procedures. A pilot study. Senses and Sciences, 3(4), 317-322.

- Guerrieri, A., Gaucher, C., Bonte, E., \& Lasfargues, J. (2012): Minimal intervention dentistry: part 4. Detection and diagnosis of initial caries lesions. British dental journal, 213(11), 551-557.

- Hibst, R., Paulus, R., \& Lussi, A. (2001): Detection of occlusal caries by laser fluorescence: basic and clinical investigations. Medical Laser Application, 16(3), 205-213.

- Jablonski-Momeni, A., Stachniss, V., Ricketts, D., HeinzelGutenbrunner, M., \& Pieper, K. (2008): Reproducibility and accuracy of the icdas-ii for detection of occlusal caries in vitro. Caries research, 42 (2), 79-87.
- Jablonski-Momeni, A., Ricketts, D. N., Heinzel-Gutenbrunner, M., Stoll, R., Stachniss, V., \& Pieper, K. (2009): Impact of scoring single or multiple occlusal lesions on estimates of diagnostic accuracy of the visual ICDAS-II system. International journal of Dentistry, (2009),1-7.

- Jablonski-Momeni, A., Schipper, H. M., Rosen, S. M., Heinzel-Gutenbrunner, M., Roggendorf, M. J., Stoll, R., Pieper, K. (2011): Performance of a fluorescence camera for detection of occlusal caries in vitro. Odontology, 99(1), $55-61$.

- Jablonski-Momeni,A., Stucke, J ., Steinberg, T., \& HeinzelGutenbrunner, M. (2012): Use of icdas-ii, fluorescencebased methods, and radiography in detection and treatment decision of occlusal caries lesions: an in vitro study. International journal of dentistry, 2012(1), 371595(1-8).

- Jablonski-Momeni, A., Liebegall, F., Stoll, R., HeinzelGutenbrunner, M., \& Pieper, K. (2013): Performance of a new fluorescence camera for detection of occlusal caries in vitro. Lasers in medical science, 28 (1), 101-109.

- Jablonski-Momeni, A., Heinzel-Gutenbrunner, M., \& Vill, G. (2016): Use of a fluorescence-based camera for monitoring occlusal surfaces of primary and permanent teeth. International journal of paediatric dentistry, 26(6), 448-456.

- Jablonski-Momeni, A., Jablonski, B., \& Lippe, N. (2017): Clinical performance of the near infrared imaging system vistacam ix proxi for detection of approximal enamel lesions. BDJ open, 3(1), 17012(1-8).

- Kingman, A. and Selwitz, R.H., (1997): Proposed methods for improving the efficiency of the DMFS index in assessing initiation and progression of dental caries. Community dentistry and oral epidemiology, 25(1), 60-68.

- Lino, J. R., Ramos Jorge, J., Coelho, V. S., Ramos Jorge, M. L., Moysés, M. R., \& Ribeiro, J. C. (2015): Association and comparison between visual inspection and bitewing radiography for the detection of recurrent dental caries under restorations. International dental journal, 65(4), 178-181.

- Marczuk-Kolada, G., Luczaj-Cepowicz, E., Obidzinska, M. and Rozycki, J., 2020. Performance of ICDAS II and fluorescence methods on detection of occlusal caries-An ex vivo study. Photodiagnosis and photodynamic therapy, 29(1), p.101609(1-6)

- $\quad$ Novaes, T. F., Matos, R., Gimenez, T., Braga, M. M., De Benedetto, M. S., \& Mendes, F. M. (2012): Performance of fluorescence-based and conventional methods of occlusal caries detection in primary molars-an in vitro study. International journal of paediatric dentistry, 22(6), 459-466. 
- Peycheva, K., \& Boteva, E. (2016): A Comparison of Different Methods for Fissure Caries Detection. Acta Medica Bulgarica, 43(1), 30-38.

- $\quad$ Presoto, C.D., Trevisan, T.C., Andrade, M.C.D., Dantas, A.A.-r., Campos, J.A.D.B., \& Oliveira-junior,O.B.D. (2017): Clinical effectiveness of fluorescence, digital images and icdas for detecting occlusal caries. Revista de Odontologia da UNESP, 46(2), 109-115.

- $\quad$ Rodrigues, J. A., Hug, I., Diniz, M. B., \& Lussi, A. (2008): Performance of fluorescence methods, radiographic examination and ICDAS II on occlusal surfaces in vitro. Caries research, 42(4), 297-304.

- Rodrigues, J. A., Diniz, M. B., Josgrilberg, É. B., \&
Cordeiro, R. C. (2009): In vitro comparison of laser fluorescence performance with visual examination for detection of occlusal caries in permanent and primary molars. Lasers in medical science, 24(4), 501-506.

- $\quad$ Seremidi, K., Lagouvardos, P., \& Kavvadia, K. (2012): Comparative in vitro validation of vistaproof and diagnodent pen for occlusal caries detection in permanent teeth. Operative dentistry, 37(3), 234-245.

- $\quad$ Stoll, R., Urban-Klein, B., Giacomin, P., Loukas, A., \& Jablonski-Momeni,A. (2015): In vivo assessment of caries excavation with a fluorescence camera compared to direct bacteriological sampling and quantitative analysis using flow cytometry. Lasers in medical science, 30 (2), 843-849 